\title{
Physical activity level in elderly assisted in an outpatient of comprehensive health care
}

\author{
Ana Tamires Jardim*, Joelma Ximenes Prado Teixeira Nascimento, Ana Tamires Jardim, \\ Joelma Ximenes Prado Teixeira Nascimento \\ From 20th Brazilian Diabetes Society Congress \\ Porto Alegre, Brazil. 11-18 November 2015
}

\section{Background}

Old age brings many changes and deleterious effects with advancing age, to minimize these declines regular physical activity has a positive effect on prevention and promotion of health of the elderly.

\section{Objective}

To identify physical activity level in elderly assisted in an outpatient of comprehensive health care.

\section{Materials and methods}

Cross-sectional study with 42 elderly treated at a clinic from a private university in São Luís-MA, conducted between the months from March to May 2015. We collected data on sociodemographic, economic, clinical and nutritional characteristics, anthropometric measurements and physical activity through the IPAQ (International Physical Activity Questionnaire). It was used the procedures of descriptive statistics and measures of association where the level of significance adopted was $p<0.05$. Data were analyzed using program Stata $12.0^{\circledR}$.

\section{Results}

The distribution between age groups showed a higher proportion of elderly between 60 and 69 yrs. (54.76\%), 78.57\% had only elementary education, married (57.14\%), retired $(52.38 \%)$ and received up to 3 minimum wages $(76.16 \%)$. Regarding physical activity, $80.95 \%$ were inactive, being more pronounced with increasing age (42.86\%), associated with this, overweight diagnosis (13.8\%), affected by hypertension (22,7\%), large medication use (47.62\%) and risk for cardiovascular diseases (23.5\%).

* Correspondence: a-tamires@hotmail.com Universidade Ceuma, São Luis, Brazil 


\begin{tabular}{|c|c|c|}
\hline Variables & $\mathbf{n}$ & $\%$ \\
\hline \multicolumn{3}{|l|}{ Idade (anos) } \\
\hline 60 a 69 & 23 & 54,76 \\
\hline$\geq 70$ & 19 & 45,24 \\
\hline \multicolumn{3}{|l|}{ Education } \\
\hline Elementary School & 33 & 78,57 \\
\hline High School & 8 & 19,05 \\
\hline Higher Education & 1 & 2,38 \\
\hline \multicolumn{3}{|l|}{ Marital status } \\
\hline married & 24 & 57,14 \\
\hline maiden & 6 & 14.29 \\
\hline separate & 3 & 7,14 \\
\hline widow & 9 & 21,43 \\
\hline \multicolumn{3}{|l|}{ Occupation } \\
\hline active & 1 & 2,38 \\
\hline Retired with activity & 3 & 7,14 \\
\hline poid & 3 & 7,14 \\
\hline unemployed & 13 & 30,95 \\
\hline Work at home without pay & 22 & 52,38 \\
\hline \multicolumn{3}{|l|}{ Income ( minimum wage) } \\
\hline up to 3 & 32 & 76,19 \\
\hline 4 to 6 & 6 & 14.29 \\
\hline 7 to 9 & 4 & 9,52 \\
\hline Above 10 & 0 & 0,00 \\
\hline \multicolumn{3}{|l|}{ Smoking } \\
\hline yes & 5 & 11.90 \\
\hline not & 37 & 88,10 \\
\hline \multicolumn{3}{|l|}{ Alcoholism } \\
\hline yes & 6 & 14,29 \\
\hline not & 36 & 85.71 \\
\hline TOTAL & 42 & 100,00 \\
\hline
\end{tabular}


clinical features characteristics of elderly assisted in an outpatient of comprehensive health care. sao luis - ma, 2015

\begin{tabular}{|c|c|c|}
\hline Variables & $\pi$ & $\%$ \\
\hline \multicolumn{3}{|l|}{ Bowel habit } \\
\hline habit normal & 14 & 33,33 \\
\hline irregular & 11 & 26,19 \\
\hline constipation & 13 & 30,95 \\
\hline diarthea & 4 & 9,52 \\
\hline \multicolumn{3}{|c|}{ Amount of drugs } \\
\hline 1 & 8 & 19,05 \\
\hline 2 to 4 & 20 & 47,62 \\
\hline$\geq 5$ & 14 & 33.33 \\
\hline \multicolumn{3}{|l|}{ Hypertension } \\
\hline yes & 28 & 66,67 \\
\hline not & 14 & 33,33 \\
\hline \multicolumn{3}{|l|}{ Diabetes } \\
\hline yes & 32 & 76,19 \\
\hline not & 10 & 23,81 \\
\hline \multicolumn{3}{|l|}{ Heart disease } \\
\hline yes & 7 & 16,67 \\
\hline not & 35 & 83.33 \\
\hline \multicolumn{3}{|c|}{ Osteoarticular diseases } \\
\hline yes & 16 & 38,10 \\
\hline not & 26 & 61,90 \\
\hline TOTAL & 42 & 100,00 \\
\hline
\end{tabular}

representation of anthropometric variables of elderly assisted in an outpatient of comprehensive health care. sao luis - ma, 2015.

\begin{tabular}{cccc}
\hline Variables & $x( \pm d p)$ & Md & $\mathbf{Q}_{q}=\mathbf{Q}_{3}$ \\
\hline Weight $(\mathrm{kg})$ & $50,63( \pm 11,79)$ & - & - \\
Height $(\mathrm{m})$ & - & 1,50 & $1,47-1,56$ \\
Body Mass Index $(\mathrm{kg} / \mathrm{m} 2)$ & $25,86( \pm 5,13)$ & - & - \\
Waist Circumference - WC $(\mathrm{cm})$ & $90,1( \pm 13,28)$ & - & - \\
Hip circumference - HC $(\mathrm{cm})$ & $90,71( \pm 11,84)$ & - \\
Reason Waist - Hip WHR $(\mathrm{cm})$ & $0,89( \pm 0,08)$ & - \\
\hline
\end{tabular}

Figure 2 Clinical feature characteristics of elderly assisted in an outpatient of comprehensive health care. Sao Luis - Ma, 2015. 
classification of elderly as the practice of physical activity according to the ipaq in . sao luis ma, 2015.

\begin{tabular}{|c|c|c|c|c|}
\hline \multirow{3}{*}{$\begin{array}{c}\text { Age } \\
\text { (years) }\end{array}$} & \multicolumn{4}{|c|}{ Classification IPAQ (minutes) } \\
\hline & \multicolumn{2}{|c|}{ Active( $\geq 150$ minutos) } & \multicolumn{2}{|c|}{ Inactive (< 150 minutos) } \\
\hline & $\mathbf{n}$ & $\%$ & $\mathbf{n}$ & $\%$ \\
\hline 60 a 69 & 7 & 16.67 & 16 & 38,10 \\
\hline$\geq 70$ & 1 & 2,38 & 18 & 42,86 \\
\hline Total & 8 & 19,05 & 34 & 80,95 \\
\hline
\end{tabular}

association between physical activity level according to demographic variables, behavioral and clinical - nutritional. sao luis ma, 2015.

\begin{tabular}{|c|c|c|c|c|c|}
\hline \multirow{3}{*}{ Variables and categories } & \multicolumn{4}{|c|}{ IPAQ (minutes) } & \multirow[b]{3}{*}{ p } \\
\hline & \multicolumn{2}{|c|}{ Active } & \multicolumn{2}{|c|}{ Inactive } & \\
\hline & $\mathbf{n}$ & $\%$ & $\mathbf{n}$ & $\%$ & \\
\hline Marital status & & & & & $p$ \\
\hline married & 6 & 4,6 & 18 & 19,4 & \multirow[t]{4}{*}{0,435} \\
\hline maiden & 0 & 0,0 & 6 & 6 & \\
\hline separate & 0 & 0,0 & 3 & 3 & \\
\hline widow & 2 & 1,7 & 7 & 7,3 & \\
\hline \multicolumn{6}{|l|}{$\begin{array}{l}\text { Body Mass Index kg / m2) } \\
\text { low weight }\end{array}$} \\
\hline low weight & 1 & 2,7 & 13 & 11,3 & \multirow[t]{4}{*}{0,476} \\
\hline eutrophic & 1 & 1,7 & 5 & 4,9 & \\
\hline overweight & 5 & 3,2 & 12 & 13,8 & \\
\hline obesity & 1 & 1,0 & 4 & 4,0 & \\
\hline \multicolumn{6}{|l|}{ Reason Waist - Hip WHR (cm) } \\
\hline cardiovascular disease risk & 6 & 5,5 & 23 & 23,5 & \multirow[t]{2}{*}{0,686} \\
\hline $\begin{array}{c}\text { Not cardiovascular disease risk } \\
\text { Amount of drugs }\end{array}$ & 2 & 2,5 & 11 & 10,5 & \\
\hline 1 & 3 & 1,5 & 5 & 6,5 & \multirow[t]{3}{*}{0,781} \\
\hline 2 to 4 & 2 & 3,8 & 18 & 16,2 & \\
\hline$\geq 5$ & 3 & 2,7 & 11 & 11,3 & \\
\hline \multicolumn{6}{|l|}{ Bowel habit } \\
\hline habit normal & 5 & 2,7 & 9 & 11,3 & \multirow[t]{4}{*}{$0,15 c$} \\
\hline irregular & 0 & 0,0 & 11 & 11 & \\
\hline constipation & 2 & 2,5 & 11 & 10,5 & \\
\hline diarrhea A & 1 & 0,8 & 3 & 3,2 & \\
\hline \multicolumn{6}{|l|}{ Hypertension } \\
\hline yes & 5 & 5,3 & 23 & 22,7 & \multirow[t]{2}{*}{0,781} \\
\hline not & 3 & 2,7 & 11 & 11,3 & \\
\hline \multicolumn{6}{|l|}{ Osteoarticular diseases } \\
\hline yes & 3 & 3.0 & 13 & 13.0 & \multirow[t]{2}{*}{0,969} \\
\hline not & 5 & 5,0 & 21 & 21,0 & \\
\hline
\end{tabular}

Figure 3 Classification of elderly as the practice of physical activity according to the IPAQ in Sao Luis Ma, 2015. 